\title{
The Effectiveness of High Intensity Laser Therapy (HILT) in the treatment of Achilles and Patellar Tendinopathy: a Systematic Review
}

\author{
Stasinopoulos Dimitrios* \\ Department of Physiotherapy, University of West Attica, Greece
}

Submission: April 14, 2021; Published: April 22, 2021

*Corresponding author: Stasinopoulos Dimitrios, Department of Physiotherapy, University of West Attica, Member of Laboratory of Neuromuscular \& Cardiovascular Study of Motion (LANECASM), Agiou Spyridonos 28, Egaleo 12243, Athens, Greece

Abstract

Purpose: The aim of the present systematic review was to determine the effectiveness of High Intensity Laser Therapy (HILT) in the management of Achilles Tendinopathy (AT) and Patellar Tendinopathy (PT) and to provide recommendations based on this evidence.

Methods: Randomized controlled trials (RCTs) identified by a search strategy in six databases were used in combination with reference checking. RCTs that included HILT, patients with AT and PT, and at least one of the clinically relevant outcome measure were selected. A qualitative analysis of the selected studies was conducted using the Furlan system.

Results: None RCTs fulfilled the inclusion criteria.

Conclusion: HILT is a dose-response modality, and the optimal treatment dose has obviously not yet have been discovered. Further research with well-designed RCTs is required to provide meaningful evidence on the effectiveness (absolute and relative) of HILT for the management of AT and PT.

Keywords: Achilles tendinopathy; Patellar tendinopathy; High intensity laser therapy; Hilt

\section{Introduction}

Achilles (AT) and Patellar (PT) Tendinopathy are the most common tendinopathies of the lower limb. The main complaints of patients with tendinopathy are pain and decreased function both of which may affect daily activities. Diagnosis is based on defining pain features (e.g. localized pain) as well as reproducing pain with specific clinical tests and palpation. Although the signs and symptoms of tendinopathy are relatively clear, to date, no ideal treatment has emerged. Many clinicians advocate a conservative approach as the treatment of choice for tendinopathy. For example, many physical therapy strategies have been proposed for the rehabilitation of tendon disorders. These include electrotherapy such as therapeutic ultrasound, extracorporeal shockwave therapy, Low Level Laser Therapy (LLLT), iontophoresis and non-electrotherapeutic modalities such as exercise, soft tissue techniques, taping and dry needling or acupuncture. These treatments aim to reduce pain and improve function in tendinopathy via different mechanism of action. Such a variety of treatment options suggests that the optimal treatment strategy is not known, and more research is needed to discover the most effective treatment in patients with tendinopathy.

The pulsed neodymium-doped yttrium aluminum garnet (Nd:YAG) laser, a form of high-intensity laser therapy (HILT), was introduced as a new treatment option in the management of tendinopathies in the last decade. HILT has a wavelength of $1064 \mathrm{~nm}$ which causes minor and slow light absorption by chromophores [1]. The superiority of HILT over LLLT is that HILT is able to reach and stimulate the larger and/or deeper areas; accordingly, during HILT therapy, significantly greater energy might be transferred into tissue compared to LLLT [2]. A large amount of HILT irradiation can be delivered to deep tissues because HILT have a shorter laser emission time and a longer laser emission interval (low duty cycle) [3]. The effectiveness of HILT in the management of AT and PT is not yet clarified. To our knowledge, there has been no review of HILT for AT and PT. Therefore, the aim of this article is to determine the effectiveness of HILT in the management of AT and PT and to provide recommendations based on this evidence. 


\section{Methods}

\section{Search strategy}

Computerized searches were performed using Medline (from 1966 to November 2020), Embase (from 1988 to November 2020), Cinahl (from 1982 to November 2020), Index to Chiropractic literature (from 1992 to November 2020), Chirolars (from 1994 to November 2020), and SportsDiscus (from 1990 to November 2020) databases. Only English language publications were considered. The following search terms were used individually or in various combinations: "patellar tendinopathy," "achilles tendinopathy," "patellar tendonitis,", "achilles tendonitis", "patellar tendinosis", "achilles tendinosis", "rehabilitation," "laser," "high power laser," "high level laser therapy," "high intensity laser" "light," "laser light," "clinical trials" and "randomised control trials." Other references were identified from existing reviews, and other papers cited in the publications were searched. Further citations from the reference sections of papers retrieved, were sought by contacting experts in the field. Others were obtained from the Cochrane Collaboration (last search November 2020), an international network of experts who search journals for relevant citations. Unpublished reports and abstracts were not considered. Keywords and search strategy were selected by the researcher only, without the help of an expert librarian with experience in searching databases to computerized health literature.

\section{Selection of studies}

To be included within the review, a study had to meet the following conditions: it had to be a randomized control trial (RCT) with or without follow-up, which included subjects aged $>18$ years old treated for AT and / OR PT. The treatment had to be any type of HILT evaluated against at least one of the following: (i) placebo; (ii) no treatment; (iii) another treatment, conservative (physical therapy intervention or medical) or operative. RCTs in which the HILT was given as part of the treatment-for example, non-steroidal anti-inflammatory drugs (NSAIDs) and HILT or ultrasound and exercise program and HILT-were excluded, because we would not know how each modality contributed to the results. However, the effectiveness of these management strategies has not been assessed in the literature. Data were sought for one of the following four primary outcome measures: pain (scales or description words), function (scales, tests, or description words), grip strength (pain-free or maximum), and a global measure (overall improvement, proportions of patients recovered, subjective improvement of symptoms).

The titles and abstracts of all studies were assessed for the above eligibility criteria. If it was absolutely clear from information provided in the title and/or abstract that the study was not relevant, it was excluded. If it was unclear from the available abstract and/or the title, the full text article was retrieved. There was no blinding to study author, place of publication, or results.
The researcher assessed the content of all full text articles, making the selection criteria.

\section{Methodological quality}

The quality of the selected papers was examined using the system proposed by Furlan et al. [4]. Each item was scored as "yes", "no", or "don't know/unsure/unclear". 'High-quality' was defined as a "yes" score of $>50 \%$. A consensus procedure was used to solve disagreement between the reviewers.

\section{Data synthesis}

A quantitative analysis of the studies was not possible due to heterogeneity of the outcome measures. Therefore, the results were summarised using a best-evidence synthesis [4]. The article was included in the best-evidence synthesis only if a comparison was made between the groups (e.g. treatment versus placebo, control or another treatment) and the level of significance was reported. The results of the study were labeled 'significant' if 1 of the 3 outcome measures on pain, function, or recovery reported significant results. The level of evidence was ranked as follows:

i. Strong evidence for effectiveness: consistently1 positive (significant) findings within multiple high-quality RCTs.

ii. Moderate evidence for effectiveness: consistently1 positive (significant) findings within multiple low-quality RCTs and/or one high-quality RCT.

iii. Limited evidence for effectiveness: positive (significant) findings within one low-quality RCT.

iv. Conflicting evidence for effectiveness: provided by conflicting (significant) findings in the RCTs $(<75 \%$ of the studies reported consistent findings).

v. No evidence found in favour of the effectiveness of the intervention: RCT(s) available, but no (significant) differences between intervention and control groups were reported.

\section{vi. No systematic review or RCT found.}

\section{Data Abstraction}

Raw data on means for all outcomes, as well as the authors' report of the study results, were extracted from the full manuscripts by the reviewer. Data on adverse events were abstracted from the studies.

Basic data were extracted including characteristics of participants (e.g., age, gender, previous treatments, and duration of disorder), outcomes (type of outcome measure and instrument) and interventions (type, dose or intensity, frequency, and duration).

\section{Results}

Zero RCTs were found to be included in this review. 


\section{Discussion}

In this review, the effectiveness of HILT was assessed by searching databases in combination with reference checking for randomized controlled clinical trials. It was the first to assess the effectiveness of HILT in the management of AT and PT. However, no RCTs were found. Therefore, it is impossible to find out the effectiveness of HILT in the management of AT and PT based on previously conducted RCTs. A form of HILT, pulsed Nd:YAG laser therapy, has been used for a variety of diseases. Effectiveness of pulsed Nd:YAG laser therapy in pain control has been shown in ankle pain [5], subacromial impingement syndrome [3], low back pain [6,7], knee osteoarthritis [8,9], plantar fasciitis [10,11] and Lateral elbow tendinopathy (LET) [12-15]. A number of previous studies have investigated the efficacy of HILT in LET, a condition similar to AT and PT. It could be recommended the HILT parameters used for the management of LET in AT and PT rehabilitation, but HILT is a dose-response modality and the optimal treatment dose (dose, frequency, duration) has obviously not yet been discovered. Moreover, a course of HILT treatment was applied in the previous LET studies in a different way based on manufacturers' claims. Therefore, future studies are needed to standardize HILT parameters in the management of tendinopathies (acute, chronic and calcific) such as LET, PT and AT.

LLLT is a treatment that has been used for more than 40 years in clinical practice for tendinopathies conditions such as AT and PT. It is primarily used in practice for pain alleviation, assisting tissue healing at cellular level and improvement of function by inference $[16,17]$. LLLT and HILT differ in their radiation characteristics. Therefore, the effects of LLLT on tendinopathies such as AT and PT cannot be translated into those for HILT.

The HILT mechanism of action is not precise. It is considered to have both photochemical and photothermal effects, which result in anti-inflammatory, anti-edema, analgesic, and restorative treatment effects [18]. The analgesic effects of HILT are thought to be based on different mechanisms of action, such as slowing the transmission of the pain stimulus and increasing the production of morphine-mimetic substances in the body [19]. In addition, it may have direct effects on nervous structures, which may increase the improvement rate of conduction blocks or inhibit transmission through the A-delta and C pain fibers [20]. This treatment provides changes in blood flow, an increase in permeability of blood vessels and accelerates the cellular metabolic response [21]. Moreover, the photochemical and photothermal effects of HILT can stimulate collagen production within the tendons, and may increase the blood flow and vascular permeability and cause anti-inflammatory effects. Thus, HILT can help repair damaged tissues and remove painful stimuli.

Methodological shortcomings of this systematic review include searching in English alone, lack of trials selection blinding, and absence of meta-analyses. However, information on the selection criteria is often missing from the methods section of an article, so blinding has to be broken to retrieve it, as there is evidence that a difference may exist between blinded and unblinded reviews. This is very time consuming, and the differences show little consistency in direction of bias or its magnitude [22]. For these reasons, we decided that the reviewer should not be blinded to the above characteristics, even though this may have increased the possibility of methodological quality and data analysis bias. Moreover, it may sometimes be difficult for reviewers to decide whether it is clinically relevant to combine the results of a group of studies in a meta-analysis-for example, studies of patients with different types of treatment, different types of comparison groups, or different clinical characteristics of patients studied. There is consensus among the editorial board of the Cochrane Back Review Group that, if relevant valid data are lacking (data are too sparse or of too low quality) or if data are statistically and clinically too heterogeneous, a meta-analysis should be avoided and reviewers should perform a qualitative review [23]. For these reasons, a qualitative review was conducted.

\section{Conclusion}

It is impossible to find out the effectiveness of HILT in the management of AT and PT because none RCTs fulfilled the inclusion criteria. HILT is a dose-response modality, and the optimal treatment dose has obviously not yet have been discovered. Therefore, further research with well-designed RCTs is required to provide meaningful evidence on the effectiveness (absolute and relative) of HILT for the management of AT and PT.

\section{References}

1. Basford JR (1995) Low intensity laser therapy: still not an established clinical tool. Lasers Surg Med 16(4): 331-342.

2. Zati A, Valent A (2006) Laser therapy in Medicine. In: Medica M (ed) Terapia Elsica: Nuove Tecnologie in Medicina Riabilitatiya, pp. 162185.

3. Santamato A, Solfrizzi V, Panza F, Tondi G, Frisardi V, et al. (2009) Shortterm effects of high-intensity laser therapy versus ultrasound therapy in the treatment of people with subacromial impingement syndrome: a randomized clinical trial. Phys Ther 89(7): 643-652.

4. van Tulder M, Furlan A, Bombardier C, Bouter L (2003) Updated method guidelines for systematic reviews in the cochrane collaboration back review group. Spine. Phila Pa 28: 1290e9.

5. Saggini R, Bellomo RG, Cancelli F (2009) Hilterapia and chronic ankle pain syndromes. Abstract from Energy for Health. Int J İnf Sci Cult 3: 37-38.

6. Fiore P, Panza F, Cassatella G, Russo A, Frisardi V, et al. (2011) Shortterm effects of high-intensity laser therapy versus ultrasound therapy in the treatment of low back pain: a randomized controlled trial. Eur J Phys Rehabil Med 47(3): 367-373.

7. Alayat MSM, Atya AM, Ali MME, Shosha TM (2014) Long-term effect of high-intensity laser therapy in the treatment of patients with chronic low back pain: a randomized blinded placebo-controlled trial. Lasers Med Sci 29(3): 1065-1073.

8. Stiglić-Rogoznica N, Stamenković D, Frlan-Vrgoc L, Avancini- Dobrović 


\section{Orthopedics and Rheumatology Open Access Journal (OROAJ)}

V, Vrbanić TS (2011) Analgesic effect of high intensity laser therapy in knee osteoarthritis. Coll Antropol 35(2): 183-185.

9. Kheshie AR, Alayat MSM, Ali MME (2014) High-intensity versus lowlevel laser therapy in the treatment of patients with knee osteoarthritis a randomized controlled trial. Lasers Med Sci 29(4): 1371-1376.

10. Ordahan B, Karahan AY, Kaydok E (2018) The effect of high-intensity versus low-level laser therapy in the management of plantar fasciitis: a randomized clinical trial Lasers Med Sci 33: 1363-1369.

11. Naruseviciute D, Kubilius R (2020) The effect of high-intensity versus low-level laser therapy in the management of plantar fasciitis: randomized participant blind controlled trial Clin Rehabil 34(8) 1072-1082

12. Dundar U, Turkmen U, Toktas H, Ulasli AM, Solak O (2015) Effectiveness of high-intensity laser therapy and splinting in lateral epicondylitis; a prospective, randomized, controlled study Lasers Med Sci 30(3): 10971107.

13. Akkurt E, Kucuksen S, Yllmaz H, Parlak S, Sallı A, et al. (2016) Long term effects of high intensity laser therapy in lateral epicondylitis patients Lasers Med Sci 31(2): 249-253.

14. Salli A, Akkurt E, Izki AA, Sen Z, Yilmaz H (2016) Comparison of High Intensity Laser and Epicondylitis Bandage in the Treatment of Lateral Epicondylitis. Arch Rheumatol 31(3): 234-238.

15. Kaydok E, Ordahan B, Solum S, Karahan AY (2019) Short-term Efficacy Comparison of High-intensity and Low-intensity Laser Therapy in the Treatment of Lateral Epicondylitis: A Randomized Double-blind Clinical Study. Arch Rheumatol 35(1): 60-67.

16. Loevschall H, Arenholt-Bindslev D (1994) Effect of low level diode laser irradiation of human oral mucosa fibroblasts in vitro. Lasers Surg Med 14(4): 347-354

17. Reddy GK, Stehno-Bittel L, Enwemeka CS (1998) Laser photostimulation of collagen production in healing rabbit Achilles tendons. Lasers Surg Med 22(5): 281-287.

18. Monici M, Cialdai F, Fusi F, Romano G, Pratesi R (2008) Effects of pulsed Nd: YAG laser at molecular and cellular level. A study on the basis of Hilterapia. Energy for Health 3: 27-33.

19. Koçyigit H, Aydemir O, Fi ek G, Olmez N, Memi A (1999) Reliability and validity of the Turkish version of short form-36 (SF-36): a study in a group of patients will rheumatic diseases. Turk J Drugs Ther 12: 102106.

20. Chow R, Armati P, Laakso EL, Bjordal JM, Baxter GD (2011) Inhibitory effects of laser irradiation on peripheral mammalian nerves and relevance to analgesic effects: a systematic review. Photomed Laser Surg 29(6): 365-381.

21. Kujawa J, Zavodnik L, Zavodnik I, Buko V, Lapshyna A, et al. (2004) Effect of low-intensity (3.75-25 J/ cm2) near-infrared $(810 \mathrm{~nm})$ laser radiation on red blood cell ATPase activities and membrane structure. J Clin Laser Med Surg 22(2): 111-117.

22. Moher D, Pham B, Jones A, Cook D J, Jadad A R, et al. (1998) Does the quality of reports of randomized controlled trials affect estimates of intervention efficacy reported in meta-analysis? Lancet 352(9128): 609-613.

23. Van Tulder MW, Assendelft WJJ, Koes BW, Bouter LM (1997) Method guidelines for systematic reviews in the Cochrane Colloboration Back review group for spinal disorders. Spine 22(20): 2323-2330.

\section{Your next submission with Juniper Publishers} will reach you the below assets

- Quality Editorial service

- Swift Peer Review

- Reprints availability

- E-prints Service

- Manuscript Podcast for convenient understanding

- Global attainment for your research

- Manuscript accessibility in different formats

( Pdf, E-pub, Full Text, Audio)

- Unceasing customer service

Track the below URL for one-step submission https://juniperpublishers.com/online-submission.php 